\title{
The transcription factor GATA3 is a downstream effector of Hoxb1
}

\section{specification in rhombomere 4}

\author{
Illar Pata ${ }^{1, \ddagger}$, Michèle Studer $2, \neq$, J. Hikke van Doorninck ${ }^{3, *}$, James Briscoe ${ }^{4}$, Sulev Kuuse ${ }^{1}$, J. Douglas Engel ${ }^{5}$, \\ Frank Grosveld ${ }^{3}$ and Alar Karis $1,3, \uparrow$ \\ ${ }^{1}$ Institute of Molecular and Cell Biology, University of Tartu, 23 Riia St, 51010 Tartu, Estonia \\ 2Department of Developmental Neurobiology, King's College London, 4th Floor, New Hunts House, Guy's Hospital, London SE1 \\ 9RT, UK \\ ${ }^{3}$ Department of Cell Biology and Genetics, Erasmus University Rotterdam, PO Box 1738, 3000 DR Rotterdam, The Netherlands \\ ${ }^{4}$ Department of Biochemistry and Molecular Biophysics, Howard Hughes Medical Institute, Columbia University, New York, \\ NY10032, USA \\ ${ }^{5}$ Department of Biochemistry, Molecular Biology and Cell Biology, North Western University, Evanston, IL 60208 USA \\ *Present address: Rudolf Magnus Institute for Neurosciences, Utrecht University, The Netherlands \\ †These authors contributed equally to this work \\ IAuthor for correspondence at address 1 (e-mail: akaris@ebc.ee)
}

Accepted 15 September; published on WWW 9 November 1999

\section{SUMMARY}

In this paper, we show that the transcription factor GATA3 is dynamically expressed during hindbrain development. Function of GATA3 in ventral rhombomere (r) 4 is dependent on functional GATA2, which in turn is under the control of Hoxb1. In particular, the absence of Hoxb1 results in the loss of GATA2 expression in $\mathrm{r} 4$ and the absence of GATA2 results in the loss of GATA3 expression. The lack of GATA3 expression in $\mathrm{r} 4$ inhibits the projection of contralateral vestibuloacoustic efferent neurons and the migration of facial branchiomotor neurons similar to
Hoxb1-deficient mice. Ubiquitous expression of Hoxb1 in the hindbrain induces ectopic expression of GATA2 and GATA3 in ventral $\mathrm{r} 2$ and $\mathrm{r} 3$. These findings demonstrate that GATA2 and GATA3 lie downstream of Hoxb1 and provide the first example of $H o x$ pathway transcription factors within a defined population of vertebrate motor neurons.

Key words: Hindbrain, Mouse, Rhombomere 4, Motor Neurons, Hoxb1, GATA2, GATA3, Regulation

\section{INTRODUCTION}

GATA3 is a member of the family of transcription factors that have recently emerged as key regulators in various developmental processes (reviewed in Simon, 1995; Molkentin et al., 1997; Koutsourakis et al., 1999). The GATA family proteins are characterised by conserved $\mathrm{C}_{4}$-type zinc finger domains that mediate binding to a DNA motif centred around the nucleotide sequence GAT (Yamamoto et al., 1990; Ko and Engel, 1993; Whyatt et al., 1993). The GATA family comprises six vertebrate members that are expressed in distinct and spatiotemporally restricted domains. Gene ablation studies have demonstrated the vital and non-redundant role of GATA factors for normal embryonic development (Pevny et al., 1991; Tsai et al., 1994; Pandolfi et al., 1995; Molkentin et al., 1997; Koutsourakis et al., 1999). GATA3-deficient mice, produced by gene targeting, die at 10-11.5 dpc (days post coitum) and exhibit severe deficits in hematopoiesis, abdominal hemorrhaging, retardation of the lower jaw, abnormal morphology of the central nervous system (CNS) (Pandolfi et al., 1995) and a block of T lymhpocyte differentiation (Ting et al., 1996; Hendriks et al., 1999). In GATA3 ${ }^{-1-}$ chimaeras, the cytoarchitecture of serotonergic neurons of the caudal raphe nuclei is affected and, as a result, they show a serious defect in their locomotor performance on a rotorod (van Doorninck et al., 1999). Altogether these findings suggest that GATA3 is involved in multiple, even unrelated, functions during development. Among all GATA genes, GATA2 and GATA3 appear to be the only family members expressed in the CNS (Yamamoto et al., 1990; Kornhauser et al., 1994; Lieuw et al., 1995) where their expression patterns overlap extensively (George et al., 1994; Kornhauser et al., 1994; Lakshmanan et al., 1999; Nardelli et al., 1999). However, at 10.5 dpc, GATA3 expression is most prominent in rhombomere 4 in the hindbrain (Nardelli et al., 1999; van Doorninck et al., 1999).

In vertebrates, the developing hindbrain becomes rostrocaudally segmented into lineage-restricted units, termed rhombomeres (r), which are crucially involved in specifying the architecture of the future brainstem. The primary candidates for conferring rhombomeric identity are Hox genes (reviewed in Lumsden and Krumlauf, 1996). Hox genes are usually expressed in domains that have diffuse posterior limits, but sharp anterior limits coincident with rhombomeric boundaries. An exception to this common profile is the rostral 
Hoxbl expression in $\mathrm{r} 4$ with sharp anterior and posterior boundaries (Murphy et al., 1989; Murphy and Hill, 1991). Differences in Hox expression profiles have their functional correlates in hindbrain development in vivo as revealed by gene targeting studies. Loss of function of Hoxal results in hindbrain segmentation defects, where $\mathrm{r} 4$ is partially deleted but its identity remains unchanged (Carpenter et al., 1993; Dolle et al., 1993). On the contrary, in the absence of Hoxbl, segmentation remains unaffected but $\mathrm{r} 4$ identity is lost resulting in abnormal migratory behaviour of $\mathrm{r} 4$-specific motor neurons (Goddard et al., 1996; Studer et al., 1996). Similarly, misexpression of Hoxal and Hoxbl results in altered hindbrain motor neuron organisation and in homeotic transformation of rhombomeres (Zhang et al., 1994; Bell et al., 1999).

A deeper understanding of the regulatory circuits that guide neuronal specification is limited by the lack of information about interactions between Hox genes and their target genes expressed in subsets of neurons. The restricted expression of GATA3 in the developing CNS led us to suspect that GATA3 might be a component of the Hoxbl-dependent genetic hierarchy specifying neuronal identities of $\mathrm{r} 4$. In this study, we show that GATA2 and GATA3 are expressed specifically in the developing $\mathrm{r} 4$ efferent system in a Hoxbl-dependent manner. Temporally, GATA2 expression precedes that of GATA3 and is required to initiate GATA3 expression in $\mathrm{r} 4$ efferent neurons. We show that GATA3 mutant mice have defects in r4-derived motor neurons similar to those found in Hoxbl mutants, providing evidence that Hoxbl exerts part of its function through GATA2 and GATA3. This is the first example of Hox pathway transcription factors within a defined population of vertebrate neurons that is not represented by any other Hox gene.

\section{MATERIALS AND METHODS}

\section{Mutant mice}

The GATA2 and Hoxb1 mutants used in these studies were previously generated by targeted disruption of the respective genes (Tsai et al., 1994; Studer et al., 1996). Generation of the GATA3 $3^{\text {nlslacZ }}$ and GATA3 $3^{\text {taulacz }}$ mice strains was as described (Hendriks et al., 1999).

For staging embryos, noon of the day of the vaginal plug observation was designated as 0.5 days post coitum (dpc). The wildtype embryos used for in situ hybridisation analysis were the $F_{1}$ progeny obtained from CVAXNMRI intercross.

\section{Whole-mount $\beta$-galactosidase procedure}

Embryos were collected in phosphate-buffered saline (PBS) and fixed for 1-2 hours in 4\% paraformaldehyde/PBS (4\% PFA) on ice. Embryos were then rinsed twice in PBS and washed $3 \times 10$ minutes in $\beta$-galactosidase buffer $(0.1 \mathrm{M}$ phosphate buffer, $\mathrm{pH} 7.3,2 \mathrm{mM}$ $\mathrm{MgCl}_{2}, 0.02 \% \mathrm{NP}-40,0.01 \%$ deoxycholate). After this, embryos were incubated in $\beta$-galactosidase buffer supplemented with $5 \mathrm{mM}$ ferricyanide, $5 \mathrm{mM}$ ferrocyanide and $1 \mathrm{mg} / \mathrm{ml}$ 5-bromo-4-chloro-3indolyl- $\beta$-D-galactopyranoside (X-gal) overnight at room temperature, with gentle rocking and protected from light. Embryos were subsequently washed $3 \times 5$ minutes in PBS and postfixed in $4 \%$ PFA overnight at $4^{\circ} \mathrm{C}$. For double-labelling with $\beta$-galactosidase and in situ hybridisation or immunohistochemistry, $\beta$-galactosidase activity was visualised before these procedures. Embryos were collected and processed through $\beta$-galactosidase staining as above, except that incubation time in staining solution was kept minimal (34 hours at $\left.37^{\circ} \mathrm{C}\right)$.

\section{In situ hybridisation and immunohistochemistry}

Whole-mount in situ hybridisation was performed as described (Wilkinson, 1993) with a few modifications, using DIG-labelled RNA probes synthesized from mouse GATA2, GATA3 and Hoxb1 cloned DNAs. For double in situ hybridisation, GATA3 probe was synthesized with digoxigenin-UTP and Hoxbl probe with fluorescein-UTP. The probes were added together to the hybridisation buffer and revealed sequentially. The anti-DIG conjugate was developed with NBT/BCIP (Boehringer), whereas the anti-FITC conjugate was revealed with Fast Red (Sigma). For photography, hindbrains were dissected out, flattened on the microscope slide and mounted with $70 \%$ glycerol in PBS. Whole-mount immunohistochemistry using the antineurofilament light chain antibody (2H3, Developmental Studies Hybridoma Bank) was done as described (Mark et al., 1993).

\section{In vivo retinoic acid (RA) treatment}

Pregnant wild-type females mated to GATA3 $3^{\text {nlslac } Z}$ heterozygous males, were administered RA at $7.5 \mathrm{dpc}$ essentially as described (Conlon and Rossant, 1992; Marshall et al., 1992). All-trans retinoic acid (Sigma) was dissolved at $25 \mathrm{mg} / \mathrm{ml}$ in DMSO and stored frozen in aliquots under argon. Just before use, a RA aliquot was diluted one in twenty in vegetable oil and $0.2-0.3 \mathrm{ml}$ delivered by gavage for a final dose of $10 \mathrm{mg} / \mathrm{kg}$ of maternal body weight. The control mice received the same mixture without RA. Embryos were recovered at $10.5 \mathrm{dpc}$ and processed for whole-mount $\beta$-galactosidase staining or in situ hybridisation as above.

\section{Generation of transgenic embryos}

The Hoxb1/ $\beta$-actin construct used in this study is the same as already described in Pöpperl et al. (1995). The DNA construct was linearised with $B a m \mathrm{HI}$ and microinjected into fertilized mouse eggs generated from crosses of $\mathrm{F}_{1}$ hybrids $(\mathrm{CBA} / \mathrm{CA} \times \mathrm{C} 57 \mathrm{BL} / 10)$. Transgenic embryos were identified by PCR using a sense oligo specific to human $\beta$-actin (5'-TATTCTCGCAGGATCAGTCG-3') and an antisense oligo specific to mouse Hoxb1 (5'-GAGAGTGCTGGGTTCTGACG$\left.3^{\prime}\right)$.

\section{Retrograde labelling}

10.5-10.75 dpc embryos were dissected in PBS and fixed in 4\% PFA. Embryos were injected with the carbocyanine dye DiI (Molecular Probes) at a final concentration of $6 \mathrm{mg} / \mathrm{ml}$ in dimethylformamide. Extensive tracer injections were made in the second branchial arch and at the r4 exit point under a dissecting microscope using a micromanipulator and a pressure microinjector. Injected embryos were kept in the dark at room temperature for one week and subsequently hindbrains were dissected free of adjacent tissue and mounted flat with the pial side uppermost. Labelling was viewed under a confocal microscope (BioRad) using a rhodamine filter. A projection of a z-series of optical sections was obtained and contrasting false colours were given using Photoshop (Adobe).

\section{RESULTS}

\section{Spatiotemporal expression pattern of GATA3 and GATA2 in the developing hindbrain}

In situ hybridisation showed that the first GATA3-expressing cells in the developing CNS appeared at $9.0 \mathrm{dpc}$, about 20somite stage, when they were confined to the ventral neural tube of the $\mathrm{r} 4$ territory (Fig. 1H). The ventral location of the signal suggested that these cells might correspond to the progenitors of the prospective $\mathrm{r} 4$ efferent neurons. GATA3 expression in the $\mathrm{r} 4$ domain increased during the next days of development (Fig. 1I,J). Additionally, another domain of GATA3-expressing cells emerged, which was located dorsal to 


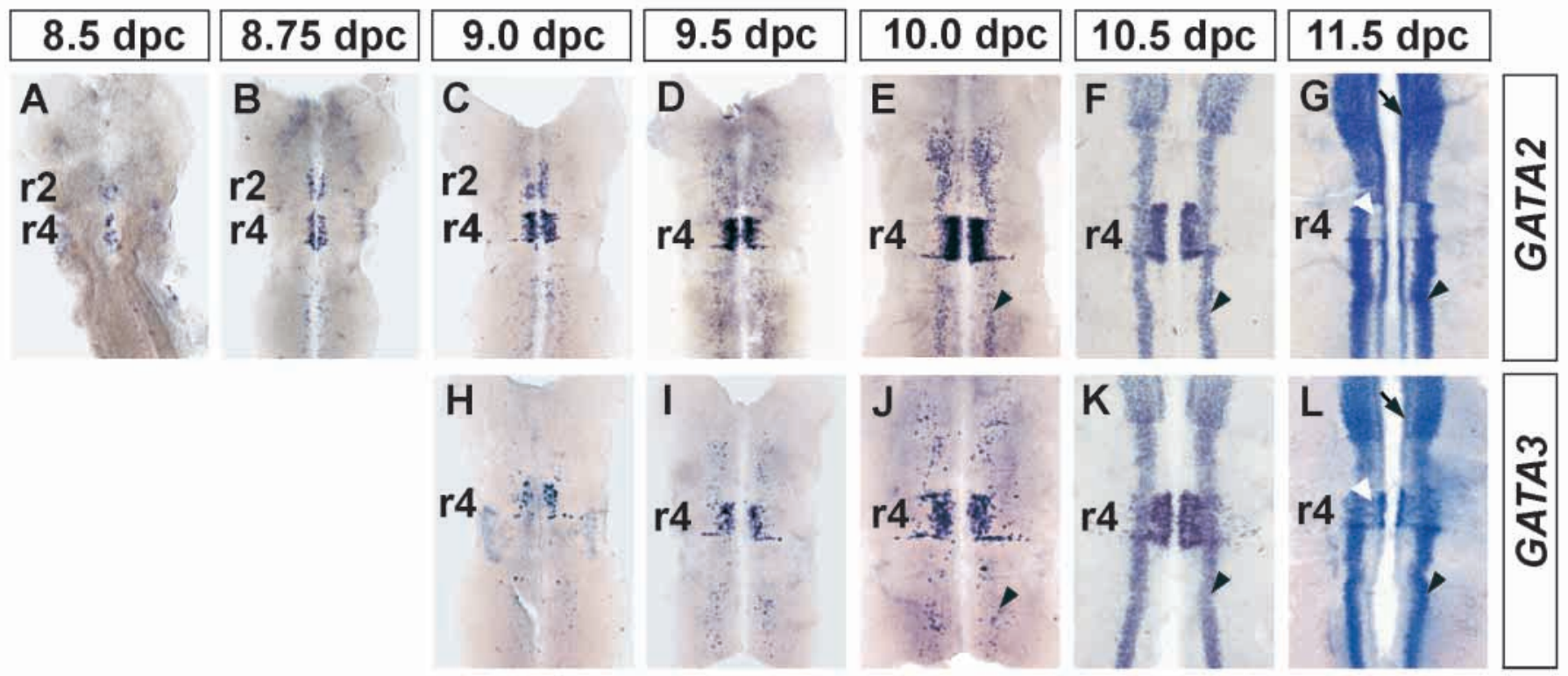

Fig. 1. Whole-mount in situ hybridisation for GATA2 (A-G) and GATA3 (H-L). Flat mounts of mouse hindbrain preparations from different embryonic stages (as indicated by dpc) are shown. Rhombomeric positions are indicated (r2, r4). Both GATA2 (A-C) and GATA3 (H) are first expressed in the ventral part of $\mathrm{r} 4$, with GATA2 expression preceding that of GATA3. Note that GATA2 is expressed also in $\mathrm{r} 2$ during 8.5-9.0 dpc. (D-F, I-K) Changes in GATA2 (D-F) and GATA3 (I-K) expression during 9.5, 10.0 and 10.5 dpc. Both GATA factors are expressed in the lateral columns that span the entire hindbrain (E,F,J,K, black arrowheads), where GATA2 expression (E,F) also precedes GATA3 (J,K). (G,L) At $11.5 \mathrm{dpc}$, GATA2 $(\mathrm{G})$ and GATA3 (L) expression is found in the contiguous ventral stripes running along the floorplate throughout the hindbrain (black arrows). Both GATA factors are expressed at high levels in the lateral columns (black arrowheads). Note that GATA3 expression persists in the ventral $\mathrm{r} 4$ domain (L, white arrowheads), whereas GATA2 expression is largely lost (G, white arrowheads).

the r4 GATA3 domain (Fig. 1J,K, black arrowhead). This population was not restricted to $\mathrm{r} 4$, but extended rostrally from the midbrain/hindbrain junction caudally to the end of the neural tube. The position and generation time of this domain was coincident with the differentiation of ventral interneurons (Ericson et al., 1997; Matise and Joyner, 1997). At 11.5 dpc,
GATA3 expression had additionally appeared in two ventral stripes that ran on either side of the floor plate through the hindbrain into the cervical spinal cord (Fig. 1L, black arrow). In r4, GATA3-expressing cells concentrated into two narrow stripes facing the floor plate on either side whereas the rest of GATA3-expressing cells were scattered along the lateral aspect
Fig. 2. Relationship of Hoxb1, GATA2 and GATA3 expression in the hindbrain. Flat-mount hindbrain preparations (rostral is up) of whole-mount in situ hybridisations to mouse embryos are shown. Rhombomere 4 position is indicated ( $\mathrm{r} 4$, bracket) to show the presence or absence of the GATA-positive ventral $\mathrm{r} 4$ domain. Arrowheads point to the GATA-positive interneuron region. (A-C) Expression of GATA2 at $10.75 \mathrm{dpc}$ in wild-type (A), $H_{0 x b 1^{-1-}}$ (B) and GATA3 $^{--}$(C) mouse embryos. (D-F) Expression of GATA3 at $10.5 \mathrm{dpc}$ in wild-type (D), $\operatorname{Hoxb1}^{-1-}(\mathrm{E})$ and GATA2 $^{-1-}(\mathrm{F})$ mouse embryos. (B,E) Expression of both GATA factors is lost in ventral $\mathrm{r} 4$ of Hoxb1 $1^{-1}$ mutants, while it persists in the interneuron region (arrowheads). (C) Expression of GATA2 is maintained in GATA $3^{-1-}$ hindbrain. (F) GATA3 expression is completely lost in ventral $\mathrm{r} 4$ and severely reduced in the interneuron region (arrowheads) in GATA2 $2^{-/-}$hindbrain.
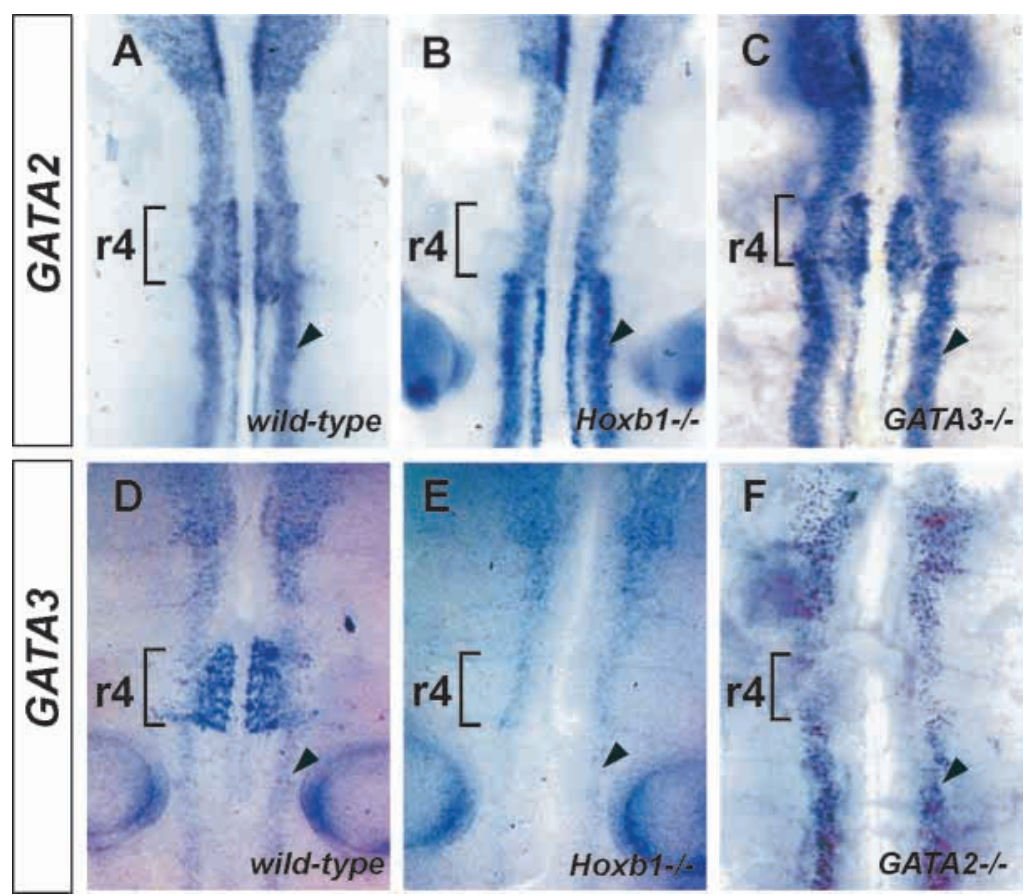
of the ventral neural tube (Fig. 1L, white arrowhead). Soma of these cells were orientated mediolaterally, indicative of ventralto-lateral migration.

Since GATA2 and GATA3 have partially overlapping expression patterns in the CNS (Kornhauser et al., 1994; Lieuw et al., 1995), we also examined GATA2 expression in the hindbrain by whole-mount in situ hybridisation. Fig. 1 shows that GATA2 expression was very similar to that of GATA3, with a few important differences. Firstly, GATA2 expression temporally preceded that of GATA3 at virtually all sites in the hindbrain. GATA2 transcript could already be detected in the ventral $\mathrm{r} 4$ domain at $8.5 \mathrm{dpc}$, just after the embryo had completed turning (12-13 somite stage) (Fig. 1A). Secondly, at early stages, GATA2 was expressed in a similar domain in $\mathrm{r} 2$ corresponding to the area that forms the trigeminal motor nucleus (Fig. 1A-C). However, during 9.5-10.5 dpc, GATA2 expression was reduced to barely detectable levels in the $\mathrm{r} 2$ domain, whereas in $\mathrm{r} 4$ its level increased dramatically (Fig. 1DF). By $11.5 \mathrm{dpc}$, GATA2 expression was downregulated in ventral r4, which continued, however, to express GATA3 (compare Fig. 1G,L). GATA2 expression also preceded that of GATA3 in the longitudinal lateral columns and in the ventral stripes adjacent to the floor plate. In contrast to the r4 domain, these sites fully retained GATA2 expression at $11.5 \mathrm{dpc}$ (Fig. $1 \mathrm{G}$ ) and at later stages of development (data not shown). Thus, it appears that GATA2 and GATA3 are expressed in a precisely controlled temporal manner. The expression domains can be classified as those being rhombomere-specific and those spanning the entire hindbrain. These domains differ in the maintenance of GATA2 expression at early stages of neuronal development: GATA2 expression is specifically lost in $\mathrm{r} 4$, whereas retained in the other domains. These results are in good accordance with those recently published by Nardelli et al. (1999), except that we observed earlier onset of expression for GATA2 as well as GATA3.

\section{GATA3 expression in $\mathrm{r} 4$ is associated with CVA neurons}

Transverse sections through the $\mathrm{r} 4$ neural tube at $10.5 \mathrm{dpc}$ showed that GATA3 was expressed in the lateral aspect of the ventral neural tube (Fig. 4A). To define more precisely the neuronal populations expressing GATA3, double-labelling with antibodies directed against GATA3 and LIM-homeodomain transcription factors was performed. These studies revealed that the ventral $\mathrm{r} 4 \mathrm{GATA} 3^{+}$cells co-expressed the motor neuron marker Isl1 whereas the GATA $3^{+}$longitudinal columns were negative for Isl 1 and formed a column of Lim $1 / 2^{+}$ventral interneurons (J. B. and I. P., unpublished; Nardelli et al., 1999). In the chick hindbrain, double-labelling of fluorescent dextran and LIM-HD markers showed that in $\mathrm{r} 4$ the lateral population of Isl1/2+ cells labelled the facial and vestibular motor neurons (Varela-Echavarria et al., 1996). Thus, the GATA3 ${ }^{+} / \mathrm{Is}_{\mathrm{s}} 1 / 2^{+}$ population in $\mathrm{r} 4$ might represent a distinct subpopulation of motor neuron precursors. Lineage tracing studies have revealed that rhombomere 4 gives rise to two major motor neuron populations: the facial branchiomotor (FBM) neurons that innervate the muscles derived from the second branchial arch and the inner ear efferent neurons (also known as contralateral vestibuloacoustic, or CVA neurons) that lead the efferent supply to the sensory fields in the inner ear. Both populations have a common origin and derive from a single motor neuron pool (Simon and Lumsden, 1993; Auclair et al., 1996; Bruce et al., 1997). During differentiation, however, the two groups display distinct migratory behaviours. The FBM neurons, which represent the largest group, migrate caudally parallel to the floor plate to form the facial motor nucleus in rostral r6, hence their perikarya are orientated rostrocaudally (Auclair et al., 1996; Studer et al., 1996; Bruce et al., 1997). Inner ear efferent neurons orientate their processes predominantly mediolaterally, migrate laterally away from the floorplate and project ipsilaterally or contralaterally across the floorplate (Fritzsch et al., 1993; Simon and Lumsden, 1993; Bruce et al., 1997).

Expression of GATA3 in $\mathrm{r} 4$ begins at $9.0 \mathrm{dpc}$ before neuronal differentiation occurs suggesting that GATA3 might be expressed in the progenitor population of the $\mathrm{r} 4$ motor neurons (Fig. 1H). However, at $10.5 \mathrm{dpc}$, a subset of the ventrally located $\mathrm{r} 4 \mathrm{GATA}^{+}$population might correspond to inner ear efferents. To test this hypothesis, we took advantage of the GATA3 ${ }^{\text {lac } Z}$ mice that were generated by gene targeting (Hendriks et al., 1999). In the GATA3taulac $Z$ and the GATA3 ${ }^{\text {nlslac } Z}$ mouse strains, $\beta$-galactosidase (E.coli lac $Z$ gene product) is expressed under the control of the GATA3 elements providing a lineage tracer for cells expressing GATA3 and, at the same time, creating a GATA3 null allele. The two strains differ in the cellular localisation of the reporter protein: in the GATA3 ${ }^{n l s l a c Z}$ mice, $\beta$-galactosidase accumulates in the nucleus of cells whereas, in the GATA3 $3^{\text {taulac } Z}$ mice, it is transported to axons and allows the visualization of axonal projections. The $\beta$-galactosidase expression pattern in the heterozygous embryos fully recapitulated that of endogenous GATA3 gene as confirmed by in situ hybridisation and immunocytochemistry (data not shown). At $10.5 \mathrm{dpc}, \beta$-galactosidase-positive neurons in $\mathrm{r} 4$ of GATA $3^{\text {taulacZ }}$ heterozygous mice formed both ipsilateral and contralateral projections, thus verifying the identity of these cells as inner ear efferents (Fig. 5A). Similar to the GATA3 antibody staining results, $\beta$-galactosidase expression was not observed in the FBM population that had already started its caudal migration into $\mathrm{r} 5$, suggesting that GATA3 is not directly involved in the migration of facial branchiomotor neurons. In summary, GATA3 is first expressed in neural progenitors located in ventral $\mathrm{r} 4$ and is subsequently maintained in the otic efferent subpopulations when they are fully differentiated (A. K. et al., unpublished data), suggesting that GATA3 might play a role in both, specification and differentiation of $\mathrm{r} 4$ efferent neurons.

\section{GATA3 expression is induced via GATA2 as a result of $H o x b 1$ expression in ventral $\mathrm{r} 4$}

The restricted expression of GATA2 and GATA 3 in ventral $\mathrm{r} 4$ during hindbrain segmentation when Hox genes are expressed in rhombomere-specific patterns led us to hypothesize that GATA genes might act downstream of Hox genes in specifying defined population of neurons. Hoxbl is initially expressed rostrocaudally along the neural tube until the presumptive $\mathrm{r} 3 / 4$ boundary and becomes restricted to $\mathrm{r} 4 \mathrm{via}$ an autoregulatory loop just before GATA2 is upregulated in $\mathrm{r} 4$ (Pöpperl et al., 1995; Studer et al., 1998). The temporal delay of GATA3 expression compared to GATA2 also suggests that GATA2 might regulate GATA3 in ventral $\mathrm{r} 4$.

We first asked whether GATA2 and GATA 3 expression in $\mathrm{r} 4$ was dependent on Hoxbl by analysing GATA expression in 
Hoxbl-deficient embryos generated by conventional gene targeting (Studer et al., 1996). In situ hybridisation showed that expression of both GATA factors were exclusively lost in ventral r4 of Hoxb1 $1^{-1-}$ embryos (Fig. 2B,E) when compared to wild-type controls (Fig. 2A,D). However, their expression was maintained in the interneuron region (indicated by a black arrowhead) and in the ventral columns adjacent to the floorplate, i.e. in the expression domains that are not restricted to $\mathrm{r} 4$. This result demonstrates that the ventral $\mathrm{r} 4$ expression of GATA2 and GATA3 is under the direct or indirect control of Hoxb1.

One prediction of such a genetic hierarchy would be that ectopic expression of Hoxbl should induce GATA expression in the hindbrain anterior to $\mathrm{r} 4$. To test this hypothesis, we first used an in vivo treatment with retinoic acid (RA) known to ectopically induce Hoxb1 expression in r2/r3 (Conlon and Rossant, 1992; Marshall et al., 1992) and next we globally overexpressed Hoxbl in transgenic mice. We gavaged RA to wild-type females mated to GATA $3^{\text {nlslac }}$ heterozygous males at day 7.5 of pregnancy and analysed the GATA3-driven $\beta$ galactosidase expression in embryos at 10.5 dpc. Fig. 3 shows that RA treatment indeed induced GATA3 expression in the ventral $\mathrm{r} 2 / \mathrm{r} 3$ territory (Fig. 3B), a domain that normally never expresses GATA3 at this stage of development (Fig. 3A). The fact that GATA3 expression pattern was controlled precisely in the same manner as Hox genes upon the RA treatment, suggests that these genes lie on the same genetic pathway. RA treatment, however, alters the expression pattern of a number of regulatory genes and therefore GATA3 might also respond directly to RA via as yet unknown regulatory elements. We therefore asked whether ectopic expression of Hoxbl alone would be sufficient to drive GATA3 expression in the same rhombomeres. It has been shown that global overexpression of Hoxbl in transgenic mice can induce its own ectopic expression in $\mathrm{r} 2 / \mathrm{r} 3$ via an autoregulatory loop (Pöpperl et al., 1995). We therefore ubiquitously expressed Hoxbl under the control of the human $\beta$-actin promoter in transgenic embryos, and analysed GATA2 and GATA3 expression at 9.5 and 10.5 dpc, respectively. Fig. 4B shows a wild-type hindbrain with Hoxbl expression in red restricted to $\mathrm{r} 4$ and GATA3 expression in blue in ventral $\mathrm{r} 4$ and in a dorsal longitudinal column as previously described. After ubiquitous expression of Hoxbl in the hindbrain, ectopic patches of GATA3 expression were detected in ventral r2 and r3 (Fig. 4C, arrows). Similarly, overexpression of Hoxbl induced higher levels of GATA2 expression in $\mathrm{r} 2$, which expanded laterally along the $\mathrm{r} 2 / \mathrm{r} 3$ boundary, a feature seen exclusively in the endogenous $\mathrm{r} 4 / \mathrm{r} 5$ boundary expression of GATA2 (Fig. 4E, arrows). The patchy ectopic pattern of GATA expression observed in these experiments might result either from insufficient levels of ectopic Hoxb1 to induce high levels of GATA2 and GATA3 expression in $\mathrm{r} 2 / \mathrm{r} 3$ or from the absence of other factors required to work in synergy with Hoxb1. Thus, in agreement with the Hoxbl loss-of-function data, Hoxbl overexpression studies confirm that GATA factors are downstream of Hoxbl in ventral $\mathrm{r} 4$, and define a regulatory cascade leading from Hoxbl to GATA genes.

We next assessed the epistatic relationship of GATA2 and GATA3 genes in $\mathrm{r} 4$ and analysed GATA2 expression in GATA3 $^{-1-}$ embryos and GATA3 expression in GATA2 ${ }^{-/-}$ embryos. Fig. 2C and $\mathrm{F}$ show that GATA2 expression was maintained in the absence of GATA3, while GATA3 expression was lost in the absence of GATA2 in the r4 ventral domain. These results are in agreement with the pattern of the onset and timing of expression of the genes and strongly suggest that GATA2 regulates GATA3 in ventral $\mathrm{r} 4$ confirming recently published data (Nardelli et al., 1999). Contrasting the results in the report mentioned above, GATA3 expression was still observed in the interneuron region of $G A T A 2^{-/-}$mutant embryos, albeit at a reduced level (Fig. 2F, arrow). Thus, although GATA2 expression also precedes GATA3 in the interneuron region, GATA3 functions, at least in part, independently of GATA2 in these cells. Taken together, our data demonstrate a regulatory cascade in ventral $\mathrm{r} 4$ that leads from Hoxb1 to GATA2 to GATA3.

\section{The migration of $\mathrm{r} 4$ efferent neurons is affected in GATA3 $^{-1-}$ embryos}

The first major defect in Hoxb1 $1^{-/-}$mutant embryos is a lack in the migration of FBM neurons into r5/r6 and of CVA neurons into the contralateral side (Goddard et al., 1996; Studer et al., 1996). Instead, motor neurons located in $\mathrm{r} 4$ express the motor neuron marker Isll and migrate into ectopic positions (Studer et al., 1996). If GATA2 and GATA3 genes are downstream effectors of Hoxbl in r4 efferent neurons, then GATA2 and GATA3 mutant embryos should reproduce at least part of the Hoxb1 mutant phenotype. A recent report on GATA2-deficient embryos (Nardelli et al., 1999) shows variable neurogenesis defects in the hindbrain including a striking reduction, but not a complete absence, of FBM neurons migrating into r5. No description was made about the behaviour of CVA neurons.

To assess whether GATA3 might be involved in the specification and/or migration of FBM and CVA neurons similarly to Hoxbl, we examined the organisation of $\mathrm{r} 4$ motor nuclei and their trajectories in GATA3 heterozygous and homozygous mutant embryos at $10.5 \mathrm{dpc}$. We first looked at GATA3 $3^{\text {nlslacZ }}$ and GATA3 $3^{\text {taulacZ }}$ heterozygous and homozygous hindbrain preparations to follow the CVA trajectory. We subsequently retrograde labelled wild-type and GATA3 ${ }^{-1-}$ mutant embryos with DiI to trace CVA and FBM trajectories.

There was no gross reduction in cell numbers and size of $\beta$-gal-positive cells in GATA3nlslacZ and GATA3taulacZ homozygous embryos, indicating that GATA3 might not be required for the early differentiation and survival of CVA neurons. However, at this early stage, there was a very severe reduction in the number of contralateral projections in GATA3 $3^{-/}$mutants (Fig. 5B,D, arrowhead) when compared to their heterozygous littermates (Fig. 5A,C), using embryos from either the GATA3 $3^{\text {taulacZ }}$ strain with $\beta$-galactosidase detection, or from the GATA3nlslacZ strain with anti-neurofilament antibody detection. Thus, as in $H o x b 1^{-1-}$ embryos, the absence of GATA3 also leads to a reduction of contralateral projections in $\mathrm{r} 4$ (Studer et al., 1996), supporting a functional relationship between Hoxbl and GATA3.

To determine whether the FBM caudal pathway was also affected in $G A T A 3^{-1-}$ mutant embryos, we retrogradely labelled the facial nerve by extensively injecting DiI in the second branchial arch along the nerve and at the $\mathrm{r} 4$ exit point in wildtype and $G A T A 3^{-1-}$ mutant embryos.

In 10.5 dpc wild-type embryos (Fig. 6A), branchiomotor cell bodies generated in ventral $\mathrm{r} 4$ initiated their caudal migration into r5 (black arrowheads), whereas CVA neurons projected 


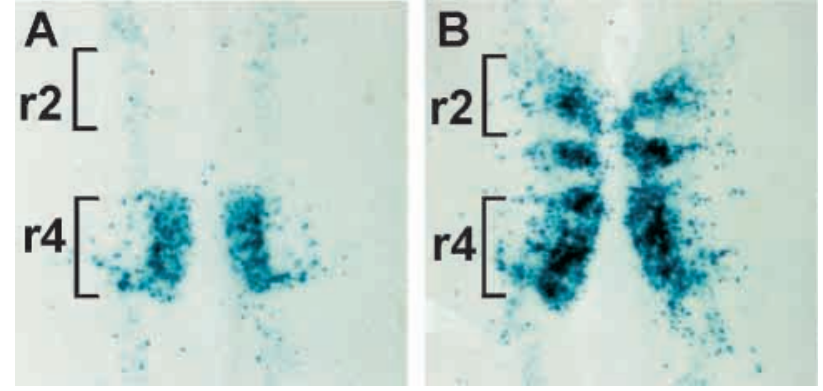

Fig. 3. Changes of GATA3 expression in the hindbrain following RA treatment. Flat-mount hindbrain preparations are shown (r2, r4 and brackets indicate rhombomere positions). $\beta$-galactosidase staining to GATA $3^{\text {nlslacZ }}$ heterozygous embryos at $10.5 \mathrm{dpc}$ treated in vivo with RA (B) or vehicle (A). Note that RA induces ectopic lacZ expression in ventral $\mathrm{r} 2 / \mathrm{r} 3(\mathrm{~B})$.

across the floor plate to the contralateral side (white arrows). In $G A T A 3^{-1-}$ homozygous mutant embryos, no facial motor neurons were seen caudal to $\mathrm{r} 4$ along the floor plate (Fig. 6B). In contrast, individual cell bodies were seen migrating laterally towards their exit points (white arrowheads) instead of caudally and/or contralaterally, a behaviour reminiscent of the affected facial neurons in Hoxb1 $1^{-1-}$ mutant embryos (Studer et al., 1996). As observed in GATA3 $3^{-1}$ embryos by $\beta$ galactosidase staining, fewer CVA neurons compared to wildtype and heterozygous embryos could be traced in homozygous mutant embryos. We also observed a reduced number of facial visceromotor neurons, normally generated in r5, albeit at variable levels (Fig. 6B, asterisk), a defect already observed in GATA2 mutant embryos (Nardelli et al., 1999).

Taken together, these results show a defect in the migration of two $\mathrm{r} 4$ efferent neuron populations, the facial branchiomotor neurons migrating into $\mathrm{r} 5$ and the vestibuloacoustic efferent neurons in the contralateral $r 4$. However, whereas the FBM migratory behaviour was completely abolished in GATA3 $3^{-1}$ mutants, the CVA projections were strongly reduced, but not eliminated. This is different from Hoxb1 $1^{--}$mutants where both populations were totally affected and suggests that either other factors are involved in this process or that GATA2 partially compensates for GATA3 function.

\section{DISCUSSION}

In this report, we have undertaken a functional analysis of the spatiotemporal expression pattern of the transcription factor GATA3 in the developing hindbrain. Our results confirm a recent report (Nardelli et al., 1999) that GATA3 is activated in ventral $\mathrm{r} 4$ after the expression of Hoxb1 and GATA2. However, our data show that in $\mathrm{r} 4$, GATA 3 is the last gene in a cascade that starts with Hoxbl and includes GATA2. We demonstrate that expression of both GATA factors is induced ectopically in Hoxbl gain-of-function embryos and abolished in Hoxbl lossof-function embryos. In addition, GATA3 expression in $\mathrm{r} 4$ is absent in GATA2 mutant embryos (our data and Nardelli et al., 1999).

\section{Relationship between GATA2 and GATA3 in the developing hindbrain}

The functions of GATA factors have been most intensively studied in hematopoiesis. GATA2 appears to be required for proliferation of hematopoietic progenitor cells and in primitive erythropoiesis, whereas GATA1 has a role in definitive erythropoiesis (Pevny et al., 1991; Tsai et al., 1994). GATA3 is essential for $\mathrm{T}$ cell development and Th2 cell differentiation (Ting et al., 1996; Zheng and Flavell, 1997; Hendriks et al., 1999). Since GATA2 is not expressed in mature hematopoietic cells (Nagai et al., 1994) and overexpression of GATA2 in an avian erythroid cell line inhibits terminal differentiation (Briegel et al., 1993), downregulation of GATA2 appears to be essential for proper hematopoietic differentiation. In contrast, studies on GATA gene expression in chick midbrain revealed that GATA2 and GATA3 were expressed in identical spatiotemporal pattern in both developing and adult optic tectum, and that variations were detected only in the relative amounts of GATA2 and GATA3 mRNAs between different cellular layers (Kornhauser et al., 1994). These data suggested that the mode of action of GATA2 in the brain might be different from that in the hematopoietic system. However, our results in

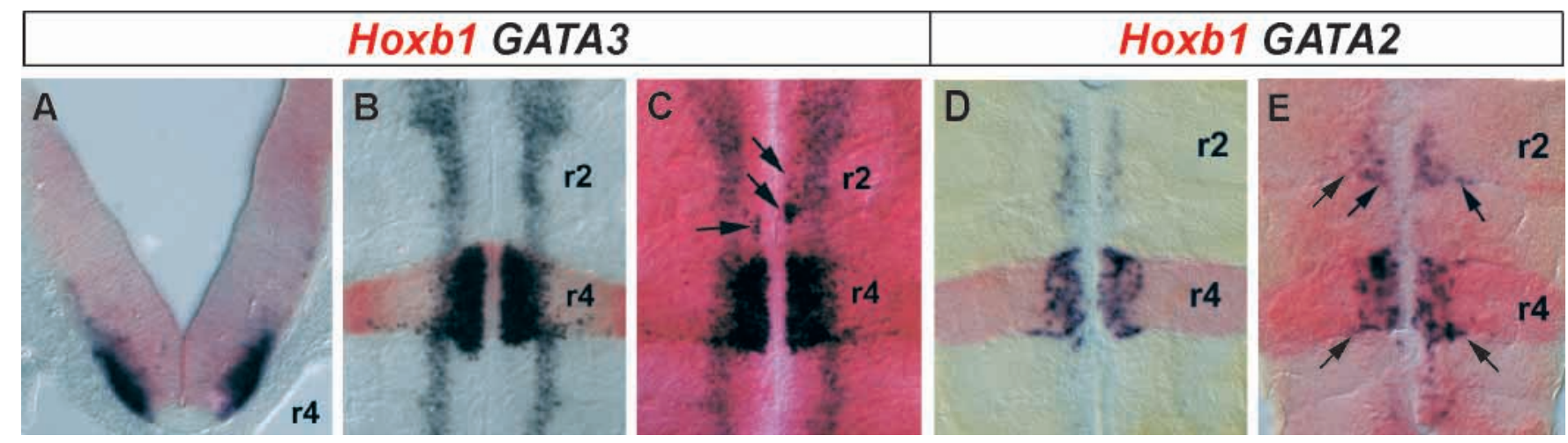

Fig. 4. Overexpression of Hoxbl in transgenic mice. Transverse section at $\mathrm{r} 4$ level (A) and flat-mount hindbrain preparations viewed from the pial surface (B-E) of $10.5 \mathrm{dpc}$ double-labelled Hoxb1/GATA3 wild-type (A,B) and transgenic embryos (C) and 9.5 dpc double-labelled Hoxb1/GATA2 wild-type (D) and transgenic embryos (E) are shown. Hoxb1 is stained in red, while GATA3 and GATA2 are visualised in dark blue. (C) Ectopic patches of GATA3-positive cells are visible in ventral $\mathrm{r} 2$ and $\mathrm{r} 3$ as indicated by arrows and (E) higher expression of GATA2 than normal is seen in $\mathrm{r} 2$. Note in E ectopic GATA2 expression expanding laterally along the boundary, a feature reminiscent to the endogenous r4 expression (arrows). 

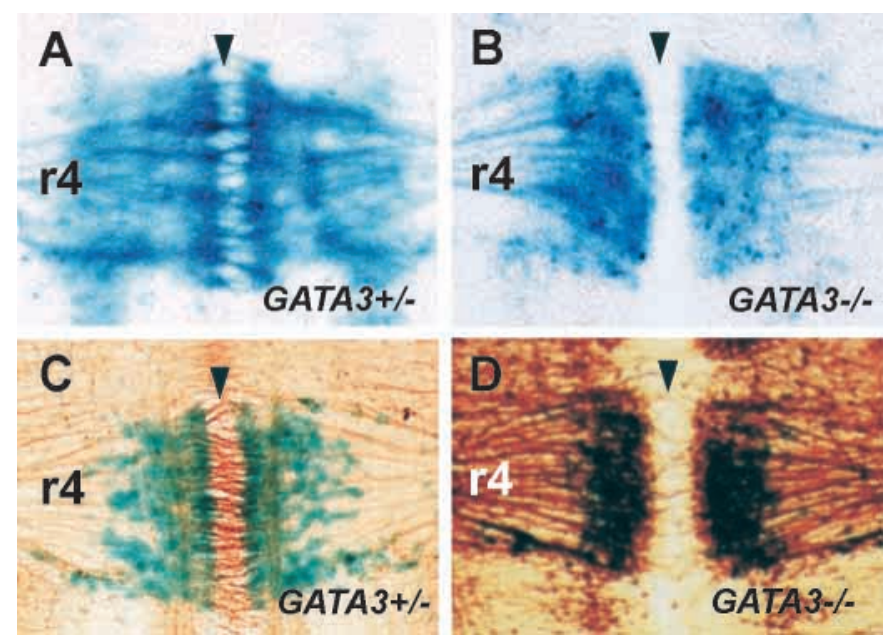

the hindbrain and at earlier phases in development, demonstrate that GATA expression in the developing brainstem follows a similar sequence of events as in hematopoietic lineages: GATA2 expression temporally precedes that of GATA3 and, at least in the $\mathrm{r} 4$ efferent neurons, GATA2 is subsequently downregulated. GATA2 function in $\mathrm{r} 4$ cannot be solely ascribed to the control of cellular proliferation, since GATA2 is also found in newly formed postmitotic neurons defined by Isl1/2 expression (Nardelli et al., 1999). Thus, GATA2 may be also involved in initial neuron maturation in $\mathrm{r} 4$. It remains currently unknown what mechanisms cause the downregulation of GATA2 in r4, and how this phenomenon is related to neuron subtype specification. It would be tempting to speculate that GATA2 is downregulated by GATA 3 and necessary to progress with the differentiation of $\mathrm{r} 4$ efferent neurons, similar to the paradigm for GATA2 function in erythropoiesis. The murine GATA3 promoter contains several consensus GATAfactor binding sites (Lieuw et al., 1997), suggesting that GATA2 might act directly on GATA3 expression in r4. In turn, GATA-sites are also present in the GATA2 promoter indicating that the transcriptional activation of GATA2 and GATA3 genes may involve crisscrossregulatory mechanisms.

Persistance of GATA2 expression in the CNS domains other than ventral $\mathrm{r} 4$ during later embryonic stages (Fig. 1G, and data not shown) suggests that GATA2 might have distinct roles in $\mathrm{r} 4$ and in interneuron/midbrain neuronal differentiation. Requirement of GATA2 for GATA3 expression also differs between these two classes of domains. In the absence of GATA2, no GATA3 expression is seen in the $\mathrm{r} 4$ ventral domain, whereas GATA3 is present at significantly reduced but still recognisable level in the interneuron region. At $10.5 \mathrm{dpc}$, GATA3-positive cells in ventral $\mathrm{r} 4$ are postmitotic, since they coexpress motor neuron marker Is $11 / 2$. Similarly to GATA3 expression in the chick optic tectum (Kornhauser et al., 1994) we did not observe a decline in GATA3 expression during mouse hindbrain development.

\section{GATA2 and GATA3 as mediator genes of Hoxb1 function in $\mathrm{r} 4$}

The observation that GATA genes were expressed in a
Fig. 5. Analysis of contralateral projections of vestibuloacoustic neurons in $G A T A 3^{-1-}$ mutant embryos. $\mathrm{r} 4$ position is indicated, arrowheads point to the floorplate. $(\mathrm{A}, \mathrm{B}) \beta$-galactosidase staining in the hindbrains of GATA $3^{\text {taulacZ }}$ embryos at $10.5 \mathrm{dpc}$. $\beta$-galactosidasepositive CVA neurons show numerous projections across the floorplate in heterozygotes (A, arrowhead), whereas only a few projections are observed in homozygous mutants (B). (C,D) $\beta$ galactosidase staining (green) and anti-neurofilament immunochemistry (brown) in hindbrains of GATA $3^{\text {nlslac }}$ embryos. (C) GATA3 ${ }^{n l s l a c Z}$ heterozygotes, showing normal nuclei of $\beta$ galactosidase-positive cells and axonal projections in r4. (D) The $\beta$ galactosidase positive nuclei appear in similar quantities, but the number of $\mathrm{r} 4$ contralaterals is decreased in GATA $3^{\text {nlslac }}$ homozygotes.

rhombomere-restricted fashion after the onset of Hox genes raised the possibility for Hox proteins to be candidate upstream regulators of GATA genes. GATA2 expression in $\mathrm{r} 4$ starts at 8.5 dpc just in a few ventral cells and GATA3 expression follows at $9.0 \mathrm{dpc}$, whereas $\mathrm{Hoxbl}$ is upregulated in $\mathrm{r} 4$ at $8.25 \mathrm{dpc}$. Among several Hox genes expressed in r4, high levels of Hoxbl are exclusive to r4 (Murphy and Hill, 1991). Hoxb1 inactivation results in altered migratory behaviour of $\mathrm{r} 4$ motor neurons, leaving the hindbrain segmentation intact (Goddard et al., 1996; Studer et al., 1996). FBM neurons in Hoxb1 $1^{-1}$ mutants initially form, but instead of migrating into r6 they remain in $\mathrm{r} 4$ and undergo a lateral migration reminiscent of that of the trigeminal motor nucleus. CVA neurons, in Hoxb1-1mutants, do not form contralateral projections. These features indicate that, in $\mathrm{Hoxb1}^{-1-}$ mutants, $\mathrm{r} 4$ might have partially adopted r2 identity (Studer et al., 1996). This study presents for the first time GATA2 and GATA3 as mediator genes of these defects. Their expression profile is specific to $\mathrm{r} 4$ at the appropriate time and, as shown by loss- and gain-of-function
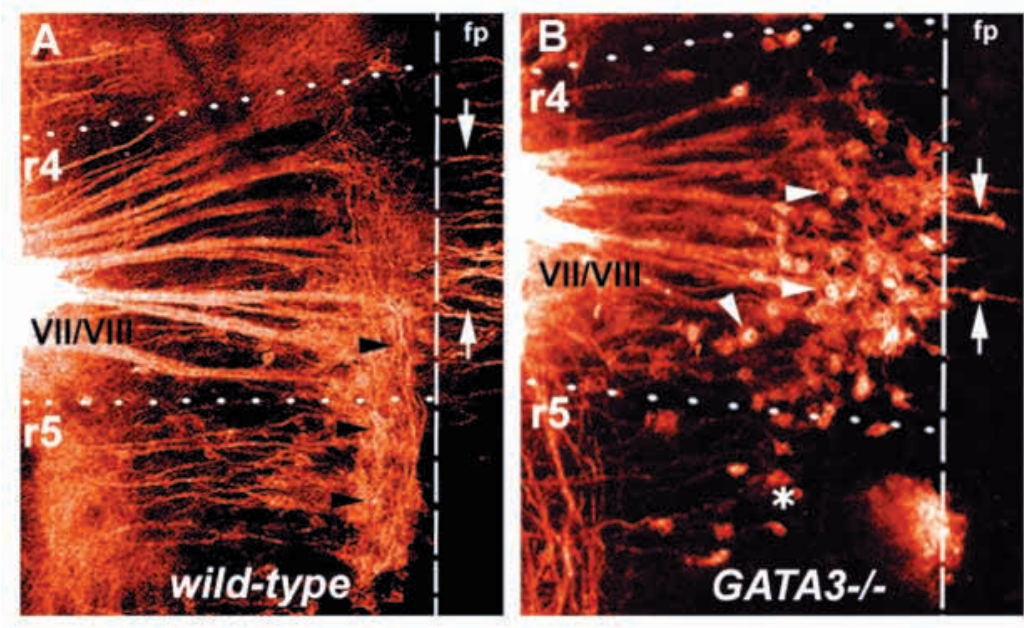

Fig. 6. Retrograde DiI labelling of the facial motor nerve in $10.5 \mathrm{dpc}$ wild-type (A) and GATA $3^{-1-}$ homozygous (B) mutant mice. DiI was injected at the VII/VIII exit point and hindbrain preparations are viewed from the pial surface. Rostral is to the top and the floor plate (fp) is to the right. (A) At $10.5 \mathrm{dpc}$ wildtype facial branchiomotor neurons (FBM) are seen in $\mathrm{r} 4$ and $\mathrm{r} 5$ (black arrowheads) and contralateral projections (CVA) have crossed the $\mathrm{r} 4$ floor plate (white arrows). GATA3 ${ }^{-1-}$ homozygous embryos (B) show a complete absence of FBM migrating caudally. The majority of cell bodies in $\mathrm{r} 4$ migrate instead laterally towards their exit point (white arrowheads). Note the dramatic reduction of both CVA crossing the floor plate (white arrows) and $\mathrm{r} 5$ facial visceromotor neurons (asterisk) compared to wild type. 
studies (Hoxb1 ${ }^{-/-}$mutant analysis and RA induction/Hoxbl overexpression experiments, respectively), r4-restricted expression of GATA factors is under the control of Hoxb1. In its early phase of expression, GATA2 is also weakly expressed in an equivalent domain in $\mathrm{r} 2$. The reason for this may lie in the notion that initially $\mathrm{r} 2$ and $\mathrm{r} 4$ are patterned in a very similar manner, and $\mathrm{r} 4$ adopts its specific identity with some temporal delay after the restriction of Hoxbl expression to r4 (Studer et al., 1996). To date, there is no evidence that Hoxb1 directly regulates GATA2. Hox/Pbx r4-specific consensus binding sites (Pöpperl et al., 1995) were not found in the human and mouse GATA2 promoter regions, for which the nucleotide sequence was available ( $600 \mathrm{bp}$ for mouse and $2200 \mathrm{bp}$ for human GATA2 gene; GenBank accession numbers AB007371 and U79137, respectively). Obviously, not all the regulatory sequences of the GATA2 locus have been defined and, therefore, the issue remains open for future studies. Alternatively, other genes involved in neurogenesis and/or neural specification might be upstream of GATA genes and be direct targets of Hoxb1. Hoxb2 $2^{-1-}$ embryos have similar facial motor nerve defects to Hoxb1 $1^{-1}$ mutants (Barrow and Capecchi, 1996; Davenne et al., 1999) and, in addition, Hoxb2 is under the direct control of Hoxbl (Maconochie et al., 1997). Therefore, Hoxb2 could be placed either between Hoxbl and GATA2 or alternatively in a parallel pathway. In the light of these results, it would be interesting to see whether GATA factors are affected in Hoxb2 mutants. Interestingly, GATA2 expression is also upregulated after global overexpression of Hoxb1 in chick embryos (Bell et al., 1999), suggesting that GATA genes are evolutionary conserved targets of Hoxbl.

Altered migratory behaviour of $\mathrm{r} 4$ motor neurons in GATA3 $^{-/-}$mutants, similar to those observed in Hoxb1 $1^{-/-}$ mutants, provides evidence for the role of GATA genes in mediating the segmental patterning clues to $\mathrm{r} 4$ motor neurons. However, the formation of contralateral projections of CVA neurons is only reduced in GATA3 ${ }^{-1-}$ mutants, instead of completely eliminated as appears to be the case in Hoxbl-1mutants. One explanation could be that the two GATA factors, which have similar DNA-binding properties, could play temporarily redundant roles. In GATA $3^{-1-}$ mutants expression levels of GATA2 are normal in ventral $\mathrm{r}$, suggesting that GATA2 could partially compensate for the loss of GATA 3 in CVA precursors. This would be similar to what has been observed in hematopoiesis, where GATA1 and GATA2 can counterbalance each other (Pevny et al., 1995). Alternatively, other factors than GATA3 may also contribute to the CVA identity.

In contrast to contralateral neurons, GATA3 appears not to be directly involved in the migration of facial branchiomotor neurons, as it is clearly not expressed in the mature FBM population during caudal migration. Instead, GATA3 appears to have a role at earlier stages in FBM development, in a precursor cell population. If $\mathrm{r} 4$ progenitor cells committed to migrate posteriorly are not properly differentiated in the absence of GATA3, then they will not be able to respond to cues responsible for migration. It is even possible that maturation of the CVA provides signals to the FBM to acquire proper specification and undergo caudal migration. However, we can not exclude that the altered migration of $\mathrm{r} 4$ motor neurons in GATA3 $^{-1}$ embryos might be due to a secondary defect, resulting from abnormalities in the CNS development not found in these studies. Clarification of these issues requires further studies, including generation of conditional knockouts, GATA2/GATA3 double knockouts and identification of GATA target genes in $\mathrm{r} 4$.

We are grateful to R. Krumlauf for the Hoxbl probe, Hoxb1-1embryos and the Hoxbl/ $\beta$-actin construct, and S. Orkin for the GATA2 mutant mouse strain. We thank I. Thesleff and H. Sariola (University of Helsinki, Developmental Biology Programme) and members of their laboratories for help, B. Fritzsch for fruitful discussions and M. Cook for technical assistance. This work was supported by EMBO fellowship (I. P.), research grants from NWO (The Netherlands; F. G, J. H. van D. and A. K.) and Estonian Science Foundation (A. K., S. K. and I. P.). M. S. is a recipient of an MRC Career Development Award.

\section{REFERENCES}

Auclair, F., Valdes, N. and Marchand, R. (1996). Rhombomere-specific origin of branchial and visceral motoneurons of the facial nerve in the rat embryo. J. Comp. Neurol. 369, 451-61.

Barrow, J. R. and Capecchi, M. R. (1996). Targeted disruption of the Hoxb2 locus in mice interferes with expression of Hoxb-1 and Hoxb-4. Development 122, 3817-3828.

Bell, E., Wingate, R. J. and Lumsden, A. (1999). Homeotic transformation of rhombomere identity after localized hoxb1 misexpression. Science 284, 2168-71.

Briegel, K., Lim, K. C., Plank, C., Beug, H., Engel, J. D. and Zenke, M. (1993). Ectopic expression of a conditional GATA-2/estrogen receptor chimera arrests erythroid differentiation in a hormone-dependent manner. Genes Dev. 7, 1097-1109.

Bruce, L. L., Kingsley, J., Nichols, D. H. and Fritzsch, B. (1997). The development of vestibulocochlear efferents and cochlear afferents in mice. Int. J. Dev. Neurosci. 15, 671-692.

Carpenter, E. M., Goddard, J. M., Chisaka, O., Manley, N. R. and Capecchi, M. R. (1993). Loss of Hox-A1 (Hox-1.6) function results in the reorganization of the murine hindbrain. Development 118, 1063-75.

Conlon, R. A. and Rossant, J. (1992). Exogenous retinoic acid rapidly induces anterior ectopic expression of murine Hox-2 genes in vivo. Development 116, 357-68.

Davenne, M., Maconochie, M. K., Neun, R., Pattyn, A., Chambon, P., Krumlauf, R. and Rijli, F. M. (1999). Hoxa2 and Hoxb2 control dorsoventral patterns of neuronal development in the rostral hindbrain. Neuron 22, 677-91.

Dolle, P., Lufkin, T., Krumlauf, R., Mark, M., Duboule, D. and Chambon, P. (1993). Local alterations of Krox-20 and Hox gene expression in the hindbrain suggest lack of rhombomeres 4 and 5 in homozygote null Hoxa1 (Hox-1.6) mutant embryos. Proc. Natl. Acad. Sci. USA 90, 7666-70.

Ericson, J., Rashbass, P., Schedl, A., Brenner-Morton, S., Kawakami, A., van Heyningen, V., Jessell, T. M. and Briscoe, J. (1997). Pax6 controls progenitor cell identity and neuronal fate in response to graded Shh signaling. Cell 90, 169-180.

Fritzsch, B., Christensen, M. A. and Nichols, D. H. (1993). Fiber pathways and positional changes in efferent perikarya of 2.5- to 7-day chick embryos as revealed with DiI and dextran amines. J. Neurobiol. 24, 1481-99.

George, K. M., Leonard, M. W., Roth, M. E., Lieuw, K. H., Kioussis, D., Grosveld, F. and Engel, J. D. (1994). Embryonic expression and cloning of the murine GATA-3 gene. Development 120, 2673-2686.

Goddard, J. M., Rossel, M., Manley, N. R. and Capecchi, M. R. (1996). Mice with targeted disruption of Hoxb-1 fail to form the motor nucleus of the VIIth nerve. Development 122, 3217-3228.

Hendriks, R. W., Nawijn, M. C., Engel, J. D., van Doorninck, H., Grosveld, F. and Karis, A. (1999). Expression of the transcription factor GATA-3 is required for the development of the earliest $\mathrm{T}$ cell progenitors and correlates with stages of cellular proliferation in the thymus. Eur. J. Immunol. 29, 1912-8.

Ko, L. J. and Engel, J. D. (1993). DNA-binding specificities of the GATA transcription factor family. Mol. Cell Biol. 13, 4011-4022.

Kornhauser, J. M., Leonard, M. W., Yamamoto, M., LaVail, J. H., Mayo, K. E. and Engel, J. D. (1994). Temporal and spatial changes in GATA transcription factor expression are coincident with development of the chicken optic tectum. Brain Res. Molec. Brain Res. 23, 100-110. 
Koutsourakis, M., Langeveld, A., Patient, R., Beddington, R. and Grosveld, F. (1999). The transcription factor GATA6 is essential for early extraembryonic development. Development 126, 723-32.

Lakshmanan, G., Lieuw, K. H., Lim, K. C., Gu, Y., Grosveld, F., Engel, J. D. and Karis, A. (1999). Localization of distant urogenital system-, central nervous system-, and endocardium-specific transcriptional regulatory elements in the GATA-3 locus. Molec. Cell Biol. 19, 1558-68.

Lieuw, K. H., Li, G. l., Zhou, Y., Grosveld, F. and Engel, J. D. (1997). Temporal and spatial control of murine GATA-3 transcription by promoterproximal regulatory elements. Dev. Biol. 188, 1-16.

Lieuw, K. H., Roth, M. E., Dzierzak, E., George, K. M., Karis, A., Leonard, M. W., Lim, K.-C., Pandolfi, P. P., Grosveld, F. and Engel, J. D. (1995). Expression and regulation of GATA-2 and GATA-3 in hematopoietic and other cell lineages. in. In Biology of Hematopoiesis and Stem Cell Gene Transfer (ed. G. Stamatoyannopoulos), pp. 15-35. Andover: Intercept Press.

Lumsden, A. and Krumlauf, R. (1996). Patterning the vertebrate neuraxis. Science 274, 1109-1115.

Maconochie, M. K., Nonchev, S., Studer, M., Chan, S. K., Popperl, H., Sham, M. H., Mann, R. S. and Krumlauf, R. (1997). Cross-regulation in the mouse HoxB complex: the expression of Hoxb2 in rhombomere 4 is regulated by Hoxb1. Genes Dev. 11, 1885-1895.

Mark, M., Lufkin, T., Vonesch, J. L., Ruberte, E., Olivo, J. C., Dolle, P., Gorry, P., Lumsden, A. and Chambon, P. (1993). Two rhombomeres are altered in Hoxa-1 mutant mice. Development 119, 319-338.

Marshall, H., Nonchev, S., Sham, M. H., Muchamore, I., Lumsden, A. and Krumlauf, R. (1992). Retinoic acid alters hindbrain Hox code and induces transformation of rhombomeres $2 / 3$ into a $4 / 5$ identity. Nature 360, 737-741.

Matise, M. P. and Joyner, A. L. (1997). Expression patterns of developmental control genes in normal and Engrailed-1 mutant mouse spinal cord reveal early diversity in developing interneurons. J. Neurosci. 17, 7805-7816.

Molkentin, J. D., Lin, Q., Duncan, S. A. and Olson, E. N. (1997). Requirement of the transcription factor GATA4 for heart tube formation and ventral morphogenesis. Genes Dev. 11, 1061-1072.

Murphy, P. and Hill, R. E. (1991). Expression of the mouse labial-like homeobox-containing genes, Hox 2.9 and Hox 1.6, during segmentation of the hindbrain. Development 111, 61-74.

Murphy, P., Davidson, D. R. and Hill, R. E. (1989). Segment-specific expression of a homoeobox-containing gene in the mouse hindbrain. Nature 341, 156-159.

Nagai, T., Harigae, H., Ishihara, H., Motohashi, H., Minegishi, N., Tsuchiya, S., Hayashi, N., Gu, L., Andres, B., Engel, J. D., and et al. (1994). Transcription factor GATA-2 is expressed in erythroid, early myeloid, and CD34+ human leukemia-derived cell lines. Blood 84, 1074-1084.

Nardelli, J., Thiesson, D., Fujiwara, Y., Tsai, F. Y. and Orkin, S. H. (1999). Expression and genetic interaction of transcription factors GATA-2 and GATA-3 during development of the mouse central nervous system. Dev. Biol. 210, 305-321.

Pandolfi, P. P., Roth, M. E., Karis, A., Leonard, M. W., Dzierzak, E., Grosveld, F. G., Engel, J. D. and Lindenbaum, M. H. (1995). Targeted disruption of the GATA3 gene causes severe abnormalities in the nervous system and in fetal liver haematopoiesis. Nat. Genet. 11, 40-44.
Pevny, L., Lin, C. S., D’Agati, V., Simon, M. C., Orkin, S. H. and Costantini, F. (1995). Development of hematopoietic cells lacking transcription factor GATA-1. Development 121, 163-172.

Pevny, L., Simon, M. C., Robertson, E., Klein, W. H., Tsai, S. F., D’Agati, V., Orkin, S. H. and Costantini, F. (1991). Erythroid differentiation in chimaeric mice blocked by a targeted mutation in the gene for transcription factor GATA-1. Nature 349, 257-260.

Pöpperl, H., Bienz, M., Studer, M., Chan, S. K., Aparicio, S., Brenner, S., Mann, R. S. and Krumlauf, R. (1995). Segmental expression of Hoxb-1 is controlled by a highly conserved autoregulatory loop dependent upon exd/pbx. Cell 81, 1031-1042.

Simon, H. and Lumsden, A. (1993). Rhombomere-specific origin of the contralateral vestibulo-acoustic efferent neurons and their migration across the embryonic midline. Neuron 11, 209-220.

Simon, M. C. (1995). Gotta have GATA. Nat. Genet. 11, 9-11.

Studer, M., Gavalas, A., Marshall, H., Ariza-McNaughton, L., Rijli, F. M., Chambon, P. and Krumlauf, R. (1998). Genetic interactions between Hoxa1 and Hoxb1 reveal new roles in regulation of early hindbrain patterning. Development 125, 1025-1036.

Studer, M., Lumsden, A., Ariza-McNaughton, L., Bradley, A. and Krumlauf, R. (1996). Altered segmental identity and abnormal migration of motor neurons in mice lacking Hoxb-1. Nature 384, 630-634.

Ting, C. N., Olson, M. C., Barton, K. P. and Leiden, J. M. (1996). Transcription factor GATA-3 is required for development of the T-cell lineage. Nature 384, 474-478.

Tsai, F. Y., Keller, G., Kuo, F. C., Weiss, M., Chen, J., Rosenblatt, M., Alt, F. W. and Orkin, S. H. (1994). An early haematopoietic defect in mice lacking the transcription factor GATA-2. Nature 371, 221-226.

van Doorninck, J. H., van der Wees, J., Karis, A., Goedknegt, E., Coesmans, M., Rutteman, M., Grosveld, F. and De Zeeuw, C. I. (1999). GATA-3 is involved in the development of serotonergic neurons in the caudal raphe nuclei. J. Neurosci. RC12, 1-8.

Varela-Echavarria, A., Pfaff, S. L. and Guthrie, S. (1996). Differential expression of LIM homeobox genes among motor neuron subpopulations in the developing chick brain stem. Mol. Cell Neurosci. 8, 242-257.

Whyatt, D. J., de Boer, E. and Grosveld, F. (1993). The two zinc finger-like domains of GATA-1 have different DNA binding specificities. EMBO J. 12, 4993-5005.

Wilkinson, D. (1993). Whole-mount in situ hybridization of vertebrate embryos. In In situ Hybridization: a Practical Approach. (ed. D. Wilkinson), pp. 75-83. Oxford: IRL Press.

Yamamoto, M., Ko, L. J., Leonard, M. W., Beug, H., Orkin, S. H. and Engel, J. D. (1990). Activity and tissue specific expression of the transcription factor NF-E1 [GATA] multigene family. Genes Dev. 4, 16501662.

Zhang, M., Kim, H. J., Marshall, H., Gendron-Maguire, M., Lucas, D. A., Baron, A., Gudas, L. J., Gridley, T., Krumlauf, R. and Grippo, J. F. (1994). Ectopic Hoxa-1 induces rhombomere transformation in mouse hindbrain. Development 120, 2431-2442.

Zheng, W. and Flavell, R. A. (1997). The transcription factor GATA-3 is necessary and sufficient for Th2 cytokine gene expression in CD4 T cells. Cell 89, 587-596. 